\title{
Artificial Immune Network Design of Optical Multiplexers/Demultiplexers
}

\author{
Carlos H. Silva-Santos, \\ São Paulo Federal Institute of Education, Science and Technology - IFSP, campus Itapetininga, 18202-000, \\ Brazil, E-mail: carlos.santos@ifsp.edu.br \\ Vitaly F. Rodríguez-Esquerre \\ Department of Electrical Engineering, Federal University of Bahia, UFBA, 40210-630, Brazil, E-mail: \\ vitaly.esquerre@ufba.br \\ Hugo E. Hernández-Figueroa \\ Department of Communications, School of Electrical and Computer Engineering, University of Campinas - \\ UNICAMP, 13082-970,Brazil,E-mail: hugo@dmo.fee.unicamp.br
}

\begin{abstract}
An efficient design approach of directional couplers based multiplexers/demultiplexers for optical communication applications, by using an adapted artificial immune network algorithm for optimization (opt-AiNet), is presented and validated by using the beam propagation method. Two key multiplexers/demultiplexers based on planar waveguides and optical fiber, directional couplers, have been optimized in order to validate the efficiency and usefulness of the opt-AiNet.
\end{abstract}

Index Terms - Immune Network Algorithm, Evolutionary Algorithms, Beam Propagation Method, Frequency Domain, Photonic Devices.

\section{INTRODUCTION}

Directional couplers (DC) are devices composed by two parallel dielectric waveguides, where the optical power transfers from one guide to another guide [1]. The coupling distance, where the maximum power transfers from one guide to another occurs, is determined by the optical and geometrical parameters of the coupler and also, by the operating wavelength [1]. They can be used as power splitters with arbitrary power fraction at the output ports, depending on the length of the coupler and their characteristics. They can also be used as two wavelength multiplexer and demultiplexer devices in wavelength division multiplexing (WDM) systems if the corresponding coupling lengths for the two wavelengths are judiciously chosen in association with the device length.

The couplers design for multiplexing/demultiplexing purposes can be considered as an inverse problem, which is usually performed by a wide number of trials, leading it to a tedious iterative process that also consume a worktime.

In [2], an artificial neural network was proposed to overcome this non trivial process, and in [3] an artificial immune network (opt-AiNet) system was adapted to optimize optical fiber directional couplers. Herein, this opt-AiNet was also extended to design novel multiplexers/demultiplexers for planar directional couplers for different CWDM wavelengths, showing the algorithm adaptation for different problems and its efficiency for these non-intuitive optimizations. 
The artificial immune network algorithm for optimization and its adaptations is presented in section II, couplers and multiplexers based in, are presented in section III, divided into planar and optical fiber based items. Optimization results are given in section IV and finally the conclusions and work in progress are presented.

\section{IMMUNE NETWORK ALGORITHM FOR OPTIMIZATION - OPT-AINET}

The Artificial Immune System (AIS) researchers and techniques have recently been expanded by the benchmarking and tackling real-world applications [4]-[10]. According to [5], its story starts on 90's, when Forrest et al. [6] proposed a selection algorithm based on the generation of T-cells to discriminate the antigen-antibody, called negative selection method. Therefore, the AIS are methaheuristics techniques being widely explored in different applications, in economy analysis [7], [8] and environmental techniques and processes improvements [9], [10].

The artificial immune network algorithm is one of the computational representation from the vertebrate immune system, being a general schematic between the antigenic and antibody relations [11], [12], [13]. Herein, is considered the number of antibodies fabricated in B-cells (Bone Marrow) to identify their geometrically complementarity, also called affinity [13].

The affinity level implies in the B-cell expansions (Figure 1), consequently, in the fabrication of new antibodies by cloning, which its can suffer some random changes (mutations) in their characteristics. It is an important concept adapted in the algorithm as important immune operator to improve the optimization performance. These new antibodies and antigenic population will be aggregated in the previous population to re-measure the affinities fees again.

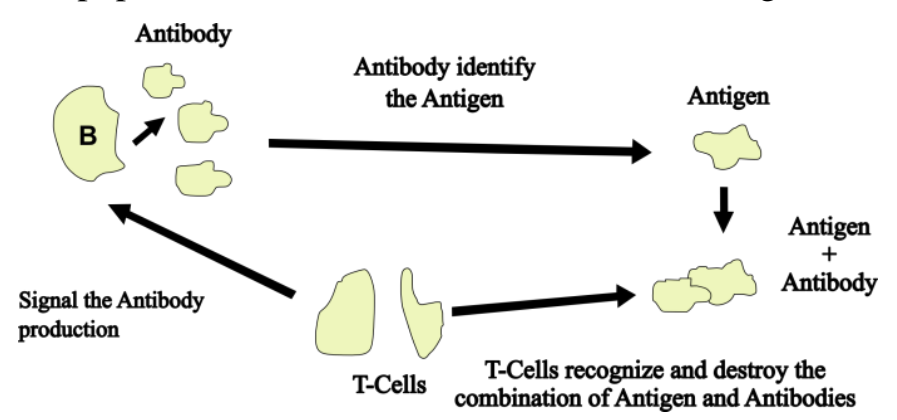

Fig. 1. Simplified vertebrate immune system schematic [3],[13].

Considering this full population, some macrophage or T-cell are signalized to suppress some antibodies/antigen, keeping in account a pre-determined region to evaluate the individuals affinities and maintain only best antibodies [11]. This complete procedure of antigenic interconnection, clonal expansion, mutation and cell selections is called clonal selection [12].

From this macro vertebrate immune system description, this work presents an adapted artificial immune system, as shown in Fig. 2, under the opt-AiNet version, combining two different mutation operators and an updated antibody suppression process to improve the convergence performance for computational electromagnetic applications. 

http://dx.doi.org/10.1590/2179-10742015v14i2442

The mutations are the combination of gene duplication and spatial Gaussian distribution [14], in proportion the proportion of $20 \%$ and $80 \%$, respectively. These relations were determined after some benchmark tests [14],[15], [16]. The suppression affinity fee was changed to consider an acceptable coupling average value for each attribute in the group of all individuals during the generation. This resource introduces flexibility for applications where the individual's attributes are pre-determined in different intervals orders of numerical values.

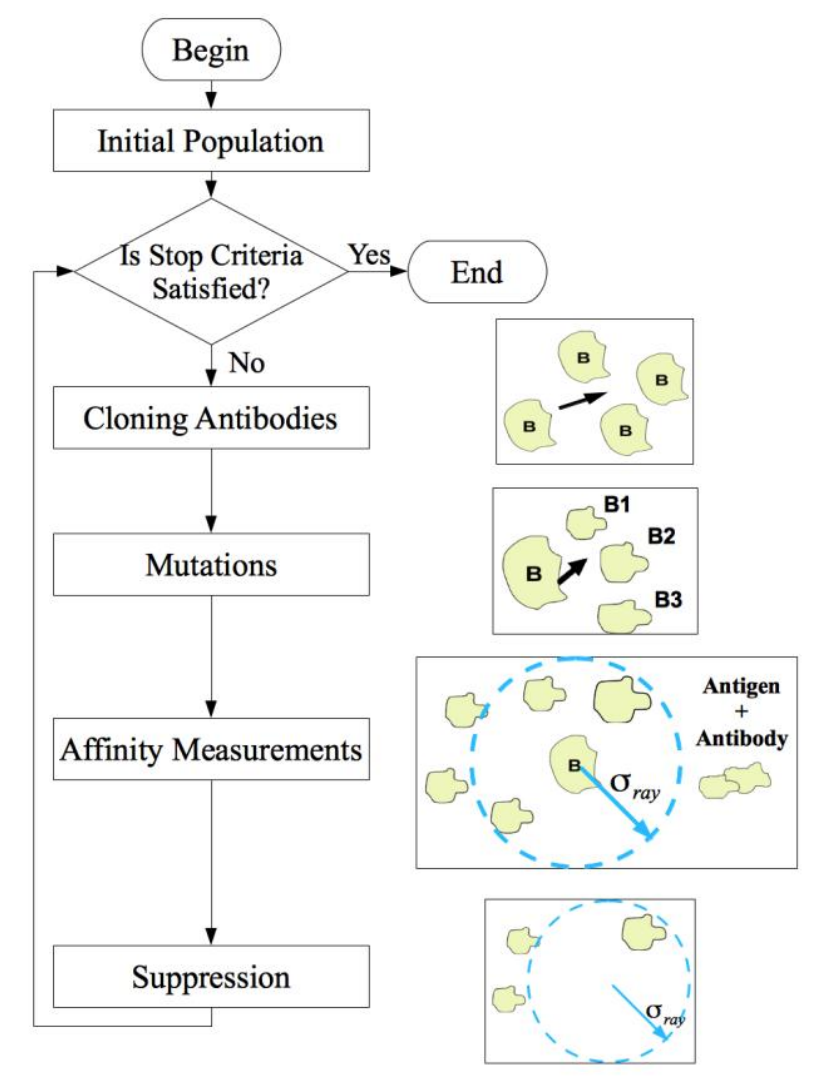

Fig. 2. Adapted Opt-AiNet algorithm dataflow.

The herein Opt-AiNet algorithm presented has been developed under the Object Orientation definitions by the $\mathrm{C}++$ language, allowing its reuse in further different applications, and follows the main theoretical aspects described in [12],[13],[17],[18]. Furthermore, this computational implementation is fast enough to be disregarded in computational powerful requirements analysis, being intrinsically related only to the objective functions. For examples, when the numerical methods, such as FDTD or FEM, are adopted to model any electromagnetic devices as presented in $0[16],[19],[20],[21]$.

\section{COUPLER BASED MULTIPLEXER}

The coupler based multiplexer/demultiplexers, as shown in Fig. 3, separate or combine two wavelength by using the directional coupler, the coupling lengths, Lc1 and Lc2, corresponding to $\lambda 1$ and $\lambda 2$, respectively, must satisfy the condition $\mathrm{L}=\mathrm{nLc} 1=\mathrm{m} \mathrm{Lc} 2$, where $\mathrm{n}$ and $\mathrm{m}$ must be odd and 
even integer numbers (or vice-versa). This ensures that each power signal will be present only in one of the outputs. The operation principle can be seen in Fig. 4 , where $n=5$ (odd) and $m=4$ (even).

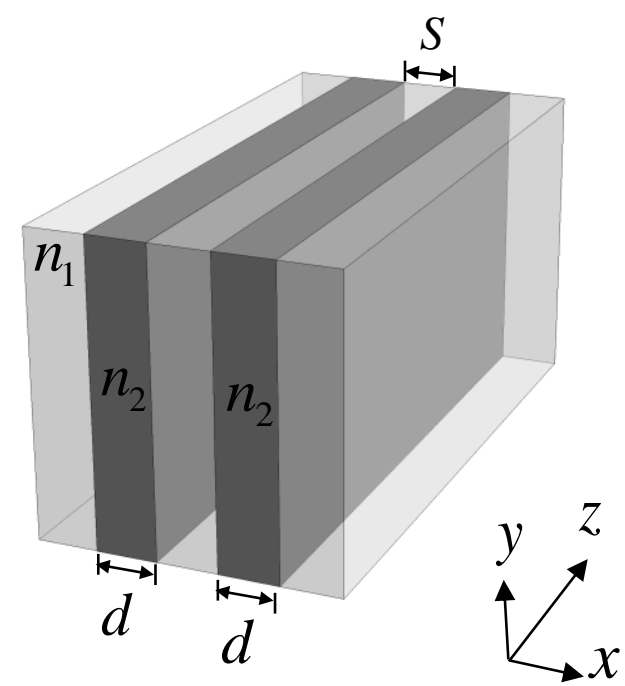

(a)

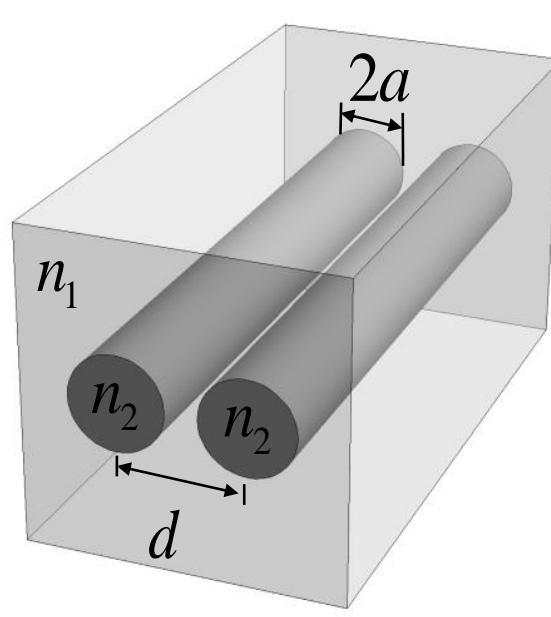

(b)

Fig. 3. Directional couplers in a) planar and b) optical fiber based waveguides.

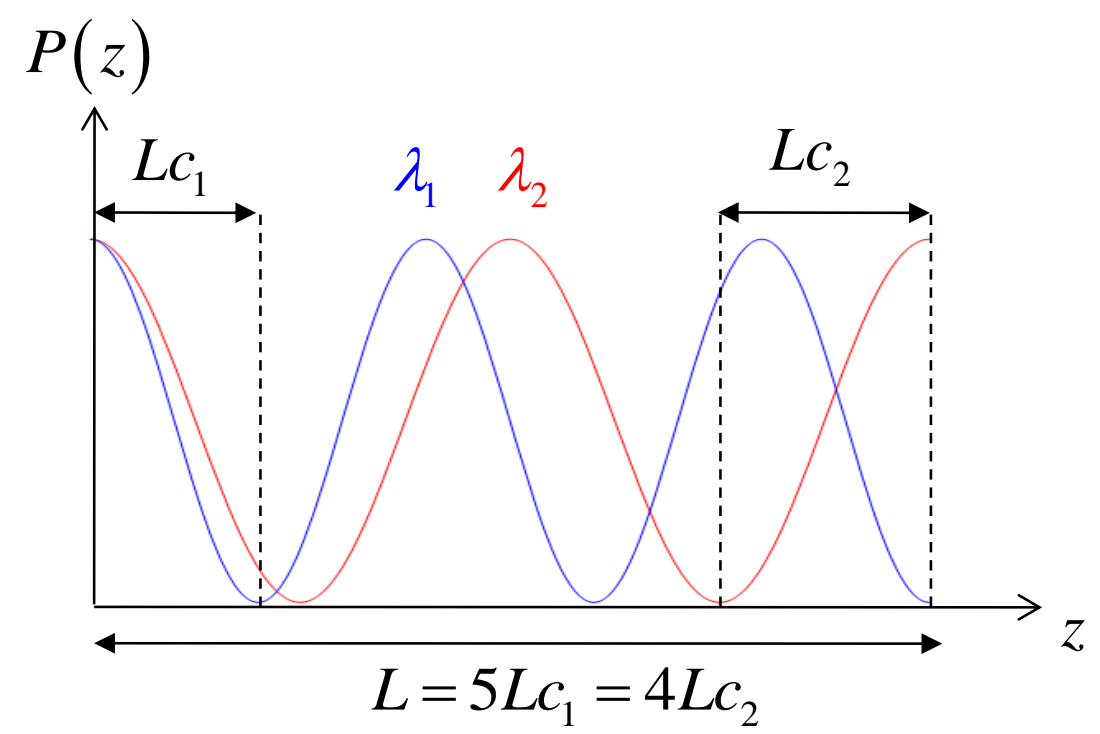

Fig. 4. Operation principles in demultiplexing directional couplers. The device length is chosen to be odd and even times the coupling length at each frequency.

These parameters are related to geometrical equations used to model the directional couplers, being important to present the Lc calculations for the planar and optical fiber directional couplers separately. At first, the planar waveguide, shown in Fig. 3. (a), operating in $\mathrm{C}$ and $\mathrm{E}$ bands. It presents the input signal along the propagation direction is given by (1) and (2),

$$
P_{1}=P_{i n} \cos ^{2}(C z)
$$



http://dx.doi.org/10.1590/2179-10742015v14i2442

$$
P_{2}=P_{i n} \operatorname{sen}^{2}(C z)
$$

where, $P 1$ and $P 2$ are the waveguide output power. $\mathrm{Pin}$ is the input power, $\mathrm{C}$ is the coupling factor obtained by the coupling mode theory and $\mathrm{z}$ is the distance measured since the input port. Therefore, the total power transfer to the other waveguide occurs when $C . z=\pi / 2$. Consequently, the coupling distance can be expressed by (3).

$$
L_{c}=\frac{\pi}{2 C}
$$

For planar waveguide DCs, the coupling factor C is given by (4),

$$
C=\frac{\alpha_{x} k_{x}^{2} e^{-\alpha_{x} S}}{k_{z}(d / 2)\left(1+2 / \alpha_{x} d\right)\left(\alpha_{x}^{2}+k_{x}^{2}\right)}
$$

where $d$ is the waveguide width, $S$ the distance between the waveguides, $k_{x}=k_{0}\left(n_{2}^{2}-n_{\mathrm{eff}}^{2}\right)^{0.5}, \alpha_{x}=k_{0}$ $\left(n_{\text {eff }}^{2}-n_{1}^{2}\right)^{0.5}$ and $k_{0}=2 \pi / \lambda$ is the wave number in free space, $\lambda$ is the wavelength, $n_{1}$ and $n_{2}$ is the guide and substrate refractive indexes, respectively. The $n_{\mathrm{eff}}$ is the effective index obtained by solving the transcendental equation (5).

$$
\tan \left(k_{x} d / 2\right)=\frac{\alpha_{x}}{k_{x}}
$$

The coupling length in optical fiber DCs shown in Fig. 2. (b) can be obtained in an approximate way by using the analytical solution given in [1],

$$
L c=\frac{\pi^{2} a^{2} n_{1} V^{2} K_{1}^{2}(W)}{\lambda U^{2} K_{0}(W d / a)}
$$

where, $\mathrm{W}=\mathrm{ak} 0($ neff2-n12)1/2, $\mathrm{U}=\mathrm{ak} 0$ (n22-neff2)1/2, $\mathrm{a}$ is the optical fiber radius, $\mathrm{d}$ is the distances between the fibers from their centers, $\mathrm{k} 0$ is the free space wavenumber given by $2 \pi / \lambda, \lambda$ is the free space wavelength, $\mathrm{n} 1$ and $\mathrm{n} 2$ are the refractive indexes of the core and the cladding, respectively. $\mathrm{K} 0$ and $\mathrm{K} 1$, are the Bessel functions.

The $n_{\text {eff }}$ is the effective index obtained by solving the transcendental equation (5).

$$
\frac{J_{0}(U)}{U J_{1}(U)}=\frac{K_{0}(W)}{W K_{1}(W)}
$$

where $J_{0}$ and $J_{l}$ are Bessel functions. We can observe the nonlinear relation among all the parameters.

\section{NUMERICAL RESULTS}

The first multiplexer/demultiplexer device designed is the planar waveguide operating in wavelengths of $1.41 \mu \mathrm{m}$ and $1.55 \mu \mathrm{m}$. The device length has been imposed as a restriction, in this way $\mathrm{L}$, should be $200 \mu \mathrm{m}$. The AIS algorithm presented satisfactory results for the convergence and for the device behavior. The parameters obtained after the optimization attended the imposed restrictions. In $\lambda=1.41 \mu \mathrm{m}$ the $\mathrm{Lc1}$ value is $66.0018 \mu \mathrm{m}$ and $\mathrm{S}=\mathrm{d}=0.538021 \mu \mathrm{m}=\mathrm{w}, \mathrm{n} 1=2.99259$ and $\mathrm{n} 2=$ 3.22757. Considering the material dispersion, in $\lambda=1.55 \mu \mathrm{m} \mathrm{n} 1=2.97971$ and $\mathrm{n} 2=3.21469$, with 
$\mathrm{Lc} 2=49.6727 \mu \mathrm{m}$. Thus, the final mux/demux proportion is $\mathrm{L} \approx 3 \cdot \mathrm{Lc} 1$ and $4 \cdot \mathrm{Lc} 2$.

In Fig. 5. it is possible to observe the propagation of the input signal which is placed at the left port, and after the propagation it can reach the left or the right port, depending on the operating wavelength.

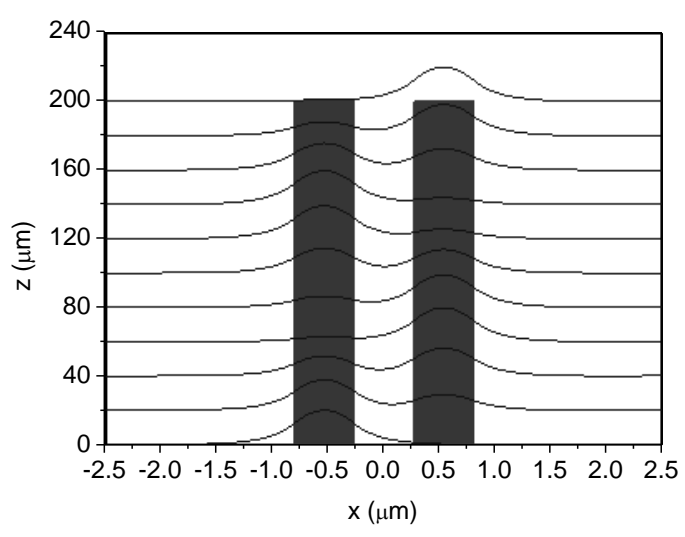

(a)

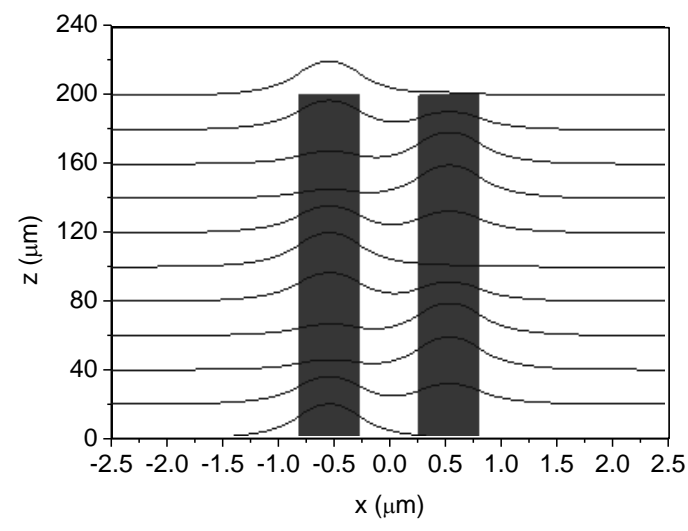

(b)

Fig. 5. Spatial distribution of the propagated fields along the designed directional coupler based on planar waveguides in (a) $\lambda=1.41 \mu \mathrm{m}$ and (b) $\lambda=1.55 \mu \mathrm{m}$.

The results obtained for the planar mux/demux are summarized in Table I.

Table I. Planar MuX/DemuX With L $=200 \mu \mathrm{m}(\mathrm{S}=\mathrm{d}=0.538021 \mu \mathrm{m})$

\begin{tabular}{ccccc}
\hline Operating Wavelength & $\mathbf{n 1}(\lambda)$ & $\mathbf{n 2}(\lambda)$ & $\mathbf{L c}(\lambda)$ & $\mathbf{L}=\mathbf{2 0 0} \boldsymbol{\mu m}$ \\
\hline$\lambda=1.41 \mu \mathrm{m}$ & 2.99259 & 3.22757 & $66.0018 \mu \mathrm{m}$ & $3.030 \mathrm{Lc}$ \\
$\lambda=1.55 \mu \mathrm{m}$ & 2.97971 & 3.21469 & $49,6727 \mu \mathrm{m}$ & $4.026 \mathrm{Lc}$ \\
\hline
\end{tabular}

The second application is the design of multiplexer/demultiplexer based on optical fiber waveguides. In this case, two CDWM wavelengths were chosen, being $\lambda 1=1.29 \mu \mathrm{m}$ and $\lambda 2=1.59$ $\mu \mathrm{m}$. The maximum device length was fixed to be $\mathrm{L}=1 \mathrm{~mm}$. The core and cladding of the optical fiber were considered to be $13.5 \% \mathrm{GeO} 2-86.5 \% \mathrm{SiO} 2$ and quenched $\mathrm{SiO} 2$, respectively.

The wavelength dependence of the refractive indexes has been taken into account by using the Sellmeier coefficients given in [22]. The values of a and d were computed by using the proposed AIS. The objective functions, for both applications, have penalties and bonuses in accordance to the limits of Lc1, Lc2, P1 and P2. For Lc1 and Lc2 the objective functions must be higher than 0 and lower than Lc limits, when these conditions are not satisfied, the final objective value is given by $f i t=-\left|L c_{1} \cdot L c_{2}\right|$

From the initial conditions, power outputs P1 and P2 promote the final objective value. When P1 and P2 have values higher than 0.98 or lower than 0.002 , the final value is given by fit $=\left(P_{1}+P_{2}\right) / 2$ and, respectively. Otherwise, it implies in.

The optimization convergence and the number of individuals are shown in Fig. 6, where the average values from ten trials are shown. For each trial, the AIS ten individuals were cloned twelve 

http://dx.doi.org/10.1590/2179-10742015v14i2442

times in each iteration (the population size was limited to 144 individuals). A mutation fee of $7 \%$ and $2 \%$ with Gaussian distribution and Genical Duplication, respectively, was used. This algorithm was executed adopting 1,000 iterations. The optimization runtime takes, in average, 29 seconds.

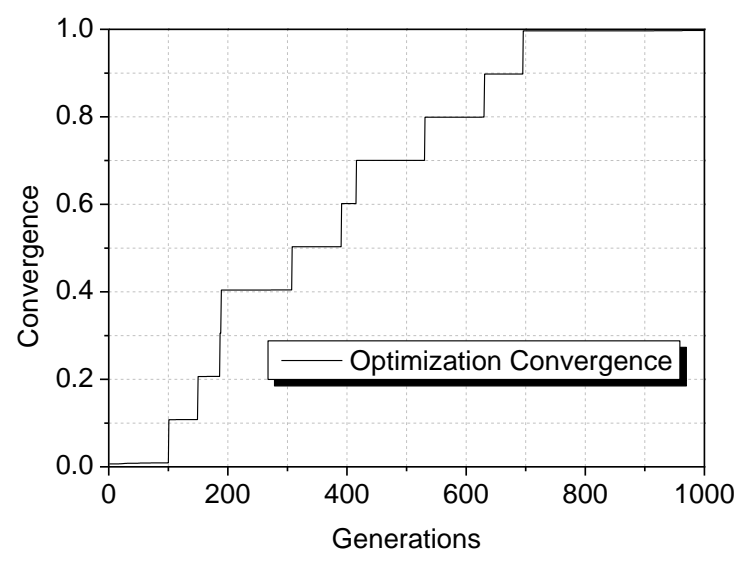

(a)

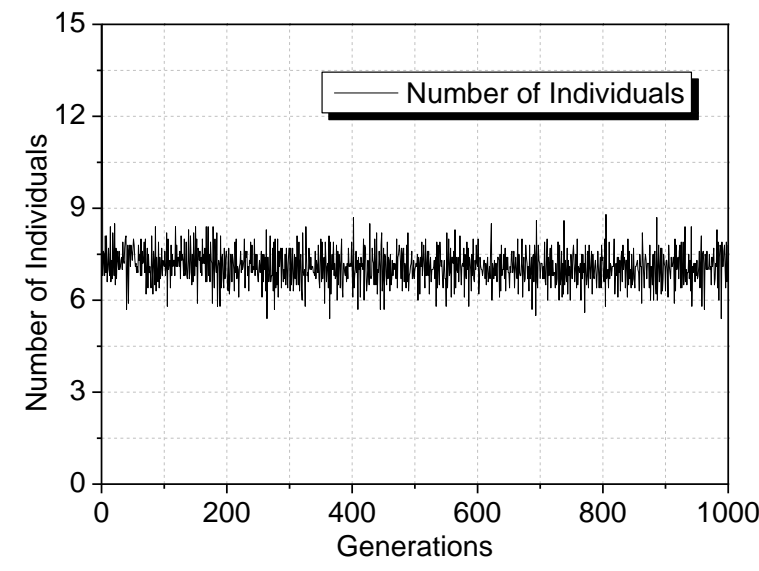

(b)

Fig. 6. The (a) convergence and (b) number of individuals in mux/demux based optical fiber waveguides.

From the optimization process with AIS the best optimized values were achieved when $\mathrm{a}=1.582$ $\mu \mathrm{m}$ and $\mathrm{d}=4.6987 \mu \mathrm{m}$, respectively, leading to coupling lengths of $\mathrm{Lc} 1=470.31 \mu \mathrm{m}$ and $\mathrm{Lc} 2=$ $314.27 \mu \mathrm{m}$ for $\lambda 1=1.29 \mu \mathrm{m}$ and $\lambda 2=1.59 \mu \mathrm{m}$, respectively. By using $\mathrm{m}=2$ and $\mathrm{n}=3$ the device length is $\mathrm{L}=941 \mu \mathrm{m}$ which is smaller than $1 \mathrm{~mm}$.

In order to validate these results, the beam propagation method in frequency domain [23] has been used to simulate the propagation of the fundamental mode along this coupler. The results are shown in Fig. 7 and 8. It can be observed that the field corresponding to $\lambda 1=1.29 \mu \mathrm{m}$ appears at the same input fiber after propagating $941 \mu \mathrm{m}$ (Fig. 7), while for $\lambda 2=1.59 \mu \mathrm{m}$ the field appears at the second fiber after propagating $941 \mu \mathrm{m}$ (Fig. 8). Another observation is that overlapping field in both cases can be considered negligible. Several other configurations have been obtained for different two CDWM wavelengths $(\lambda 1, \lambda 2, \mathrm{Lc} 1, \mathrm{Lc} 2, \mathrm{~m}, \mathrm{n}, \mathrm{L})$ and presented satisfactory results.

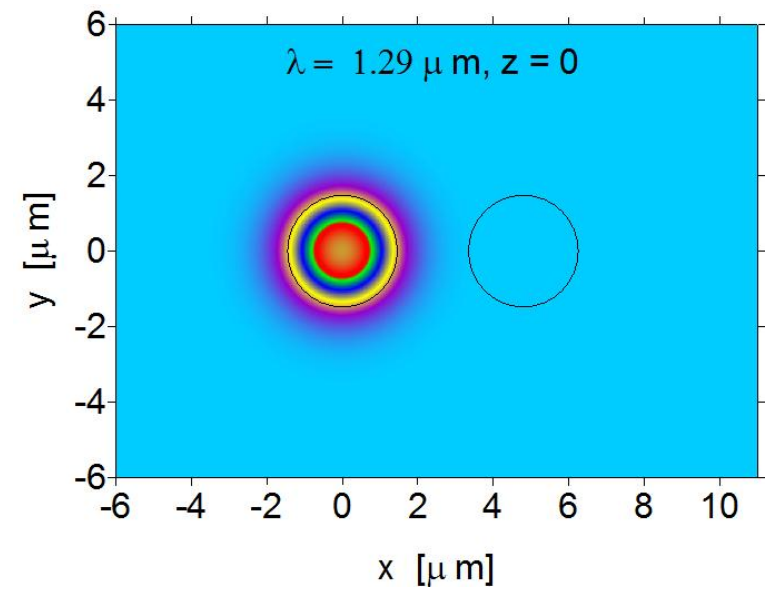

(a)

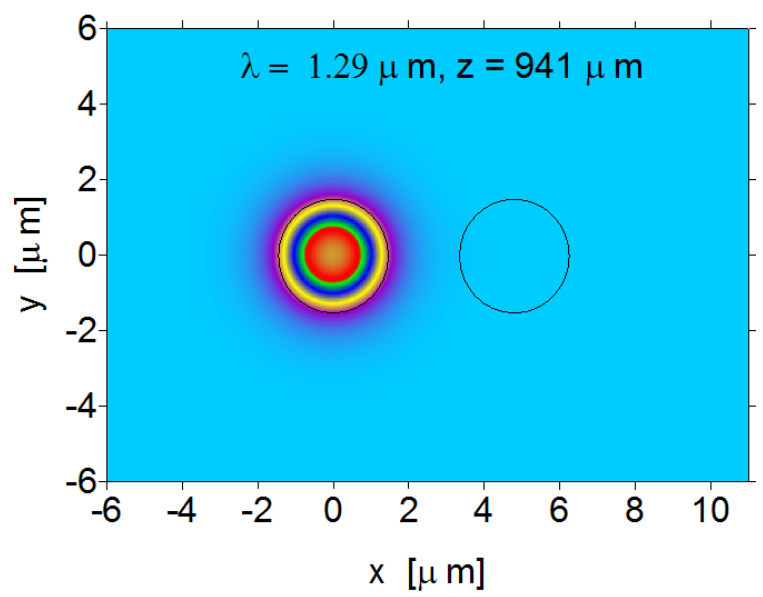

(b) 

http://dx.doi.org/10.1590/2179-10742015v14i2442

Fig. 7 Spatial distribution of the field intensity at the input and output of the coupler for $\lambda_{1}=1.29 \mu \mathrm{m}$ (a) $\mathrm{z}=0$ and (b) $\mathrm{z}=$ $941 \mu \mathrm{m}$.

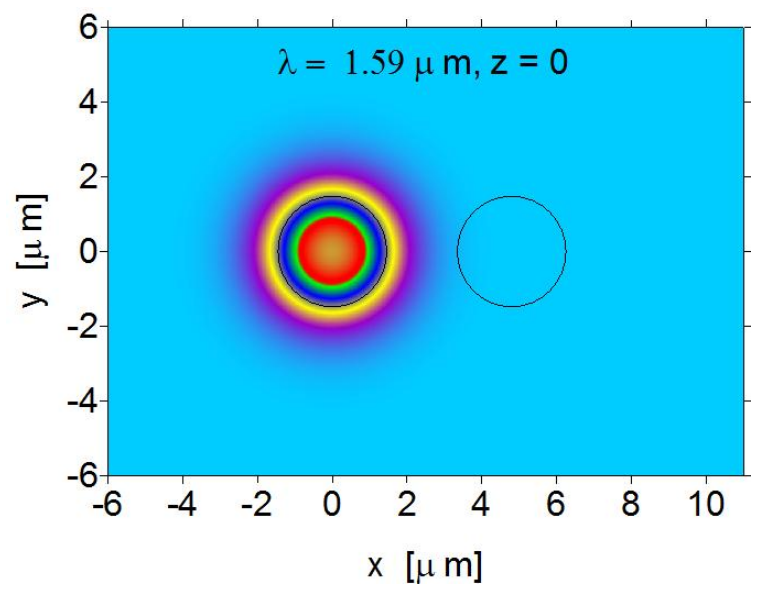

(a)

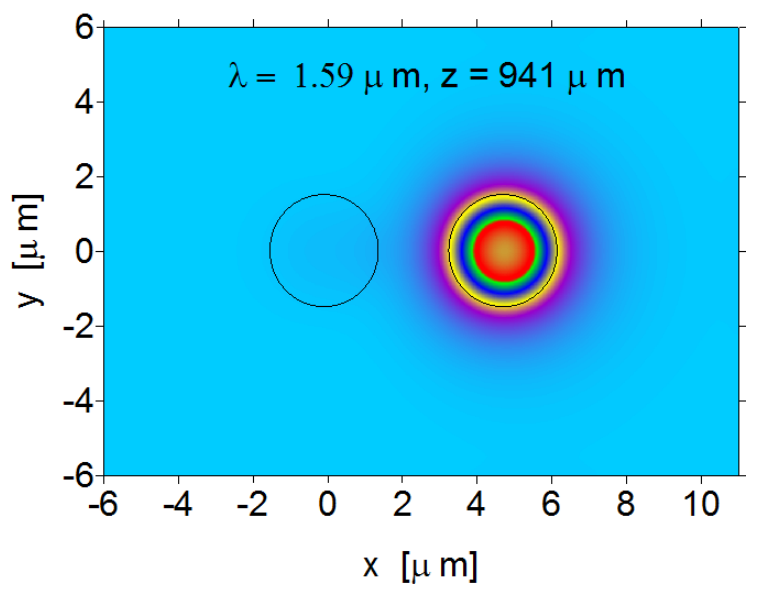

(b)

Fig. 8 Spatial distribution of the field intensity at the input and output of the coupler for $\lambda_{2}=1.59 \mu \mathrm{m}(\mathrm{a}) \mathrm{z}=0$ and (b) $\mathrm{z}=$ $941 \mu \mathrm{m}$.

The results obtained for the optical fiber mux/demux are summarized in Table II.

TABle II. Optical Fiber MuX/Demux with L $=941 \mu \mathrm{m}(\mathrm{a}=1.582 \mu \mathrm{m}$ and $\mathrm{d}=4.6987 \mu \mathrm{m})$

\begin{tabular}{ccccc}
\hline Operating Wavelength & $\mathbf{n 1}(\lambda)$ & $\mathbf{n 2}(\lambda)$ & $\mathbf{L c}(\lambda)$ & $\mathbf{L}=\mathbf{9 4 1} \boldsymbol{\mu m}$ \\
\hline$\lambda=1.29 \mu \mathrm{m}$ & 1.4683 & 1.4474 & $470.31 \mu \mathrm{m}$ & $2.0008 \mathrm{Lc}$ \\
$\lambda=1.59 \mu \mathrm{m}$ & 1.4651 & 1.4439 & $314.27 \mu \mathrm{m}$ & $2.994 \mathrm{Lc}$ \\
\hline
\end{tabular}

\section{CONCLUSIONS}

The use of efficient parallel platforms and algorithms may certainly help to reduce substantially the CPU time. In this work, the AIS in conjunction with analytical formulae have been used to design planar and optical fiber couplers based CDWM multiplexers/demultiplexers in an efficient way. The optimizations presented here showed a satisfactory convergence, optimized results with the constraints imposed and short runtime. The results were validated by using the FEM-BPM.

\section{ACKNOWLEDGMENTS}

The authors thank CePOF/UNICAMP/FAPESP, INCT FOTONICOM/CNPq/FAPESP, FAPESP (under the processes 2005/55958-8 and 2006/57074-2) and CNPq under the process 311774/2012-1, CAPESP, FAPESB.

\section{REFERENCES}

[1] K. Okamoto, Fundamentals of Optical Waveguides, Academic Press, 2005.

[2] V. F. Rodríguez-Esquerre, A. Dourado-Sisnando, and F. G. S. Silva, "Neural Network Analysis and Design of Directional Couplers," in Integrated Photonics Research, Silicon and Nanophotonics and Photonics in Switching, OSA Technical Digest (CD) (Optical Society of America, 2010), paper JTuB25. 

http://dx.doi.org/10.1590/2179-10742015v14i2442

[3] C. H. Silva-Santos, V. F. Rodríguez-Esquerre, and H. E. Hernández-Figueroa, "An Artificial Immune System for Optical Fiber Based Directional Couplers Multiplexer/Demultiplexers Design," in Latin America Optics and Photonics Conference, OSA Technical Digest (CD) (Optical Society of America, 2010), paper PDPTuJ5.

[4] E. Hart, Emma; J. Timmis. "Application areas of AIS: The past, the present and the future.” Applied soft computing, v. 8, n. 1, p. 191201, 2008.

[5] D. Dasgupt; S. Yu; F. Nino. Recent advances in artificial immune systems: models and applications. Applied Soft Computing, v. 11, n. 2, p. 1574-1587, 2011

[6] S. Forrest, A.S. Perelson, L. Allen, R. Cherukuri, Self-nonself discrimination in a computer, IEEE Symposium on Research in Security and Privacy, Los Alamitos, CA, 1994.

[7] M. Basu. Artificial immune system for combined heat and power economic dispatch. International Journal of Electrical Power \& Energy Systems, v. 43, n. 1, p. 1-5, 2012.

[8] M. Shukla; S. Jharkharia. An inventory model for continuously deteriorating agri-fresh produce: an artificial immune system-based solution approach. International Journal of Integrated Supply Management, v. 9, n. 1, p. 110-135, 2014.

[9] O. Erdinc; M. Uzunoglu. Optimum design of hybrid renewable energy systems: overview of different approaches. Renewable and Sustainable Energy Reviews, v. 16, n. 3, p. 1412-1425, 2012.

[10] S. Li, et al. Self-adaptive obtaining water-supply reservoir operation rules: Co-evolution artificial immune system. Expert Systems with Applications, v. 41, n. 4, p. 1262-1270, 2014

[11] L. N. de Castro, "Fundamentals of Natural Computing: Basic Concepts, Algorithms, and Applications, Chapman \& Hall/CRC, 2006.

[12] L. De Castro, Fundamentals of Natural Computing: An Overview, Physics of Life Reviews (4) 1-36, 2007

[13] L. N. de Castro, J. Timmis, “Artificial Immune Systems: A New Computational Intelligent Approach”, Springer Verlag, London, 2002.

[14] C. H. Silva-Santos, M. S. Gonçalves, H. E. Hernández-Figueroa, "Designing Novel Photonic Devices by Bio-Inspired Computing", IEEE Photonics Technology Letter, Vol. 22, Issue 15, 2010.

[15] C. H. Silva-Santos, K. Claudio, M. S. Gonçalves, J. R. Brianeze, H. E. Hernández-Figueroa, "Bio-Inspired Algorithms Applied to Microstrip Antennas Design", Journal of Computational Interdisciplinary Science, Vol. 1, Issue 2, 2009.

[16] J. R. Brianeze, C. H. Silva-Santos, H. E. Hernández-Figueroa. "Multiobjective evolutionary algorithms applied to microstrip antennas design algoritmos evolutivos multiobjetivo aplicados a los proyectos de antenas microstrip." Ingeniare. Revista chilena de ingeniería 17, 3, 288-298, 2009.

[17] J. Timmis, A. Hone, T. Stibor, E. Clark, "Theoretical advances in artificial immune systems". Theoretical Computer Science, 403(1), $11-32,2008$

[18] D. Dasgupta, S. Yu, F. Nino, "Recent advances in artificial immune systems: models and applications." Applied Soft Computing, 11(2), 1574-1587, 2011.

[19] A. D. Sisnando, et al. "Artificial immune system optimisation of complete bandgap of bidimensional anisotropic photonic crystals." IET Optoelectronics 9.6 (2015): 333-340.

[20] A. D. Sisnando, et al. "Power Coupling Optimization by Artificial Immune System." Integrated Photonics Research, Silicon and Nanophotonics. Optical Society of America, 2014.

[21] C. H. Silva-Santos, H. E. Hernandez-Figueroa. "Parallel bio-inspired algorithms in computational electromagnetics applications." 2011 SBMO/IEEE MTT-S International Microwave and Optoelectronics Conference (IMOC 2011). 2011.

[22] M. Adams, An Introduction to Optical Waveguides, Wiley, 1986.

[23] Y. Tsuji and M. Koshiba, "Finite element beam propagation method with perfectly matched layer boundary conditions for threedimensional optical waveguides,” Int. J. Numer. Modeling: Electronic Networks, Devices and Fields, vol. 13, pp. 115-126, 2000. 